\title{
Vers une science de la soutenabilité ?
}

Après avoir constaté l'usage extensif et abusif de certains concepts dans le domaine des sciences de l'environnement, le précédent éditorial de NSS en appelait à davantage de réflexivité. Les exemples cités reposaient sur des notions à la mode. Mais le regard doit aussi se porter sur des concepts plus anciens, qui connaissent d'importantes évolutions, comme c'est le cas pour celui de développement durable, avec l'émergence d'une "science de la soutenabilité ». En quelques années, entre 2005 et 2010, pas moins de cinq revues internationales à comité de lecture ont, en effet, été lancées pour promouvoir cette approche et en rendre compte : Sustainability: Science, Practice, \& Policy; Sustainability Science ; une section dédiée à la sustainability science dans les Proceedings of the National Academy of Sciences; Sustainability: The Journal of Record ; Consilience: The Journal of Sustainable Development. Cette intense activité éditoriale n'est qu'une illustration parmi d'autres de la volonté qui s'est manifestée au tournant du siècle de bâtir une «nouvelle science » centrée sur le thème de la soutenabilité. Elle s'est accompagnée de la création de nombreux enseignements consacrés à cette science ; de l'édition de nombreux ouvrages et manifestes; de l'organisation de colloques périodiques, soutenus par diverses organisations internationales, dont l'Unesco, l'International Council for Science (ICSU), le Programme des Nations unies pour l'environnement (PNUE) ou même, plus récemment, la Commission européenne... Tout cela témoigne de la préoccupation, qui a pris sa source à la fin des années 1990, à la fois aux États-Unis et dans plusieurs institutions scientifiques engagées dans la problématique du changement global, de replacer sur un terrain scientifique la thématique du développement durable, menacée de devenir un "slogan » sans portée réelle. C'est une dynamique extrêmement forte qui a sa géographie et une organisation en réseau spécifique, concentrées autour de quelques grandes universités nord-américaines (Harvard, Yale, Berkeley, Michigan), des Pays-Bas ou scandinaves (Lund, Karlskrona...), du Japon (Tokyo...) et surtout de quelques grands instituts (International Institute for Applied Systems Analysis
[IIASA], Stockholm Environment Institute, Resilience Institute, Santa Fe Institute, Université des Nations unies...) ou programmes scientifiques internationaux (International Human Dimensions Programme of Global Environmental Change [IHDP]...). Comme on peut le constater, la France y est relativement peu présente ${ }^{1}$.

Pendant longtemps, ce sont essentiellement les économistes néoclassiques de la croissance et des ressources naturelles et les chercheurs travaillant dans la mouvance de l'économie écologique qui se sont efforcés de conceptualiser ce qu'était le développement durable. C'est en 1989 qu'ont été créées l'International Society for Ecological Economics (ISEE) et la revue Ecological Economics. Les scientifiques engagés dans cette entreprise étaient essentiellement des économistes et des écologues, notamment ceux qui, comme Robert Costanza, élève d'Howard Odum, développaient une approche des écosystèmes centrée sur l'analyse écoénergétique. D'emblée, comme en témoigne le titre de l'ouvrage édité après le premier colloque de l'ISEE organisé à Washington en 1990, l'économie écologique entendait - elle aussi - être la science de la soutenabilité ${ }^{2}$ alors que l'économie néoclassique restait assez méfiante vis-à-vis de cette notion. Le positionnement de l'économie écologique a conduit à mettre au centre de cette problématique la question de la valeur des services écosystémiques; de leur possible - ou impossible - substituabilité technique par des dispositifs produits par les hommes ; et de leur commensurabilité par rapport aux échelles de valeur instituées par la société, notamment monétaires. Même si l'ambition multidisciplinaire était - et est encore - clairement revendiquée, la problématique demeure essentiellement de créer une "nouvelle économie » censée appréhender simultanément l'économie de la nature et l'économie des hommes.

\footnotetext{
1 Pour un état des lieux en Europe, voir Jäger, J., 2009. Sustainability science in Europe, European Commission, DG Research.

2 Costanza, R. (Ed.), 1991. Ecological economics. The science and management of sustainability, New York, Columbia University Press.
} 
Les promoteurs de la "science de la soutenabilité » - parmi lesquels on peut citer William Clark, Robert Kates et Jill Jäger - ont, à première vue, une vision plus large des disciplines à mobiliser pour promouvoir un développement durable, puisque toutes les sciences de la nature et de la société doivent a priori y participer. Dans la pratique, néanmoins, l'accent est surtout mis sur la compréhension fondamentale des dynamiques d'interaction entre systèmes naturels et systèmes humains dans une perspective de soutenabilité ${ }^{3}$. Les textes fondateurs de cette «nouvelle discipline» partent de l'écologie, des sciences de la Terre et des questions de risque et de vulnérabilité, pour défendre une approche holiste et systémique des relations entre système terrestre et société, ce qui les conduit à prendre en compte les sciences humaines dans une approche plutôt comportementaliste, complémentaire des démarches des "sciences dures ». Au centre de cette problématique, se trouvent l'analyse des interfaces, souvent à l'échelle terrestre ou de grandes régions écologiques, entre les systèmes humains et les systèmes naturels ainsi que les questions de pressions, de limites, d'adaptation, de résilience... Implicite ou explicite, la référence aux travaux, qui datent des années 1970, de C.S. Holling sur la résilience des systèmes écologiques est constante. Et les réflexions s'inspirent fortement de l'ouvrage fondateur publié par l'IIASA, un an avant le rapport Brundtland, sous la direction de W.C. Clark et R.E. Munn ${ }^{4}$. Les thématiques privilégiées sont ainsi celles du changement global et de la gestion des ressources vivantes (agriculture, conservation des sols, gestion des pêches et des forêts, biodiversité), avec une attention beaucoup plus limitée portée à l'industrie, la ville, la gestion locale, les inégalités ou les modes de vie ${ }^{5}$. L'approche est moins sociologique ou économique que systémique avec une place importante donnée aux indicateurs et à la modélisation prospective. En résumé, beaucoup des interrogations qui sont au cœur de la problématique de la soutenabilité y sont abordées, mais elles sont loin d'être les seules possibles. Comme c'est le cas pour l'économie écologique, la perspective reste, en définitive, elle aussi, spécifique.

3 Clark, W.C., Dickson, N.M., 2003. Sustainability science, the emerging research program, Proceedings of the National Academy of Sciences, 100, 14, 8059-8061. Voir aussi Kates, R.W., et al., 2001. Sustainability science, Science, 292, 5517, 641-642.

4 Clark, W.C., Munn, R.E. (Eds), 1986. Sustainable development of the biosphere, Cambridge, Cambridge University Press.

5 Kajikawa, Y., 2008. Research core and framework of sustainability science, Sustainability Science, 3, 215-239. Voir aussi Kates, R.W., 2011. What kind of a science is sustainability science?, Proceedings of the National Academy of Sciences, 108, 49, 19449-19450.
L'économie écologique, les sciences de la soutenabilité - on pourrait en citer d'autres - constituent autant d'approches et de formes d'intégration disciplinaire indispensables pour penser et évaluer le développement durable. Peut-on pour cela parler d'une science du développement durable? Le colloque "La notion de durabilité : quelles pistes pour la recherche ? », organisé en 1997 par Natures Sciences Sociétés-Dialogues ${ }^{6}$, avait bien montré qu'aucune science spécifique ne peut s'attribuer le monopole de l'étude du développement durable et que, si toutes peuvent y contribuer utilement, aucune, non plus, ne peut éviter qu'il subsiste dans les notions de développement et de soutenabilité une part de valeurs et de choix sociopolitiques qui n'est pas réductible à une norme scientifique. Il peut y avoir une science de la gestion des ressources naturelles et des limites écologiques - même si l'on en connaît les difficultés pratiques de mise en œuvre - mais la notion de développement durable est beaucoup plus large et ouverte, avec des problèmes politiques et sociaux de choix de développement, souvent mal pris en compte dans les approches précédentes. Si l'on peut partager la crainte mise en avant par les promoteurs de la sustainability science d'un affadissement de la rhétorique du développement durable, au moment où celle-ci tend à disparaître du débat public ${ }^{7}$, il n'est pas sûr, malheureusement, que l'affirmation de cette nouvelle science suffira à lui redonner toute la légitimité nécessaire.

Sans préjuger de l'avenir du concept de développement durable, NSS ne pouvait pas se désintéresser de ce mouvement scientifique majeur à l'interface entre sciences de la société et sciences de la nature. C'est ce qui est fait dans ce numéro avec l'interview d'une de ses personnalités aujourd'hui les plus reconnues au niveau international, Katherine Richardson ${ }^{8}$.

Jacques Theys et Franck-Dominique Vivien

\footnotetext{
6 Il a donné lieu à la publication d'un ouvrage : Jollivet, M. (Ed.), 2001. Le développement durable, de l'utopie au concept, Paris, Elsevier.

7 Voir le dossier publié en octobre 2013 et février 2014 dans la revue Développement durable et territoires : "Le développement durable, concept sous-exploité ou idée dépassée ? », avec des articles de F.-D. Vivien, E. Zaccaï et J. Theys.

8 Voir aussi Berdoulay, V., Soubeyran, O., 2014. Adaptation, science de la durabilité et pensée planificatrice, Natures Sciences Sociétés, 22, 2, 114-123.
} 